Article

\title{
Vitamin D3 Metabolites Demonstrate Prognostic Value in EGFR-Mutant Lung Adenocarcinoma and Can be Deployed to Oppose Acquired Therapeutic Resistance
}

\author{
Tatiana Shaurova ${ }^{1}$, Grace K Dy ${ }^{2}$, Sebastiano Battaglia ${ }^{3}$, Alan Hutson ${ }^{4}$, Letian Zhang ${ }^{1}$, \\ Yunkai Zhang ${ }^{5}$, Christine M Lovly ${ }^{5}$, Mukund Seshadri ${ }^{6}{ }^{(0)}$, David W Goodrich ${ }^{1}$, \\ Candace S Johnson ${ }^{1}$ and Pamela A Hershberger ${ }^{1, *}$ (D) \\ 1 Department of Pharmacology and Therapeutics, Roswell Park Comprehensive Cancer Center, Buffalo, \\ NY 14263, USA; tatiana.shaurova@roswellpark.org (T.S.); letian.zhang@roswellpark.org (L.Z.); \\ david.goodrich@roswellpark.org (D.W.G.); candace.johnson@roswellpark.org (C.S.J.) \\ 2 Department of Medicine, Roswell Park Comprehensive Cancer Center, Buffalo, NY 14263, USA; \\ Grace.Dy@roswellpark.org \\ 3 Center for Immunotherapy, Roswell Park Comprehensive Cancer Center, Buffalo, NY 14263, USA; \\ sebastiano.battaglia@roswellpark.org \\ 4 Department of Biostatistics and Bioinformatics, Roswell Park Comprehensive Cancer Center, Buffalo, \\ NY 14263, USA; alan.hutson@roswellpark.org \\ 5 Department of Medicine and Vanderbilt Ingram Cancer Center, Vanderbilt University Medical Center, \\ Nashville, TN 37232, USA; yunkai.zhang@vanderbilt.edu (Y.Z.); christine.lovly@vanderbilt.edu (C.M.L.) \\ 6 Department of Oral Oncology, Roswell Park Comprehensive Cancer Center, Buffalo, NY 14263, USA; \\ mukund.seshadri@roswellpark.org \\ * Correspondence: pamela.hershberger@roswellpark.org; Tel.: +1-716-845-1697
}

Received: 27 January 2020; Accepted: 9 March 2020; Published: 13 March 2020

\begin{abstract}
EGFR tyrosine kinase inhibitors (EGFR TKIs) are the standard of care treatment for patients with EGFR-mutant lung adenocarcinoma (LUAD). Although initially effective, EGFR TKIs are not curative. Disease inevitably relapses due to acquired drug resistance. We hypothesized that vitamin D metabolites could be used with EGFR TKIs to prevent therapeutic failure. To test this idea, we investigated the link between serum 25-hydroxyvitamin D3 (25(OH)D3) and progression-free survival (PFS) in patients with EGFR-mutant LUAD that received EGFR TKIs (erlotinib $\mathrm{n}=20$ and afatinib $\mathrm{n}=1$ ). Patients who were 25(OH)D3-sufficient experienced significantly longer benefit from EGFR TKI therapy (mean 14.5 months) than those with 25(OH)D3 insufficiency (mean 10.6 months, $p=0.026$ ). In contrast, $25(\mathrm{OH}) \mathrm{D} 3$ had no prognostic value in patients with KRAS-mutant LUAD that received cytotoxic chemotherapy. To gain mechanistic insights, we tested 1,25-dihydroxyvitamin D3 (1,25(OH)2D3) activity in vitro. 1,25(OH)2D3 promoted epithelial differentiation and restored EGFR TKI sensitivity in models of EGFR TKI resistance that were associated with epithelial-mesenchymal transition (EMT). 1,25(OH)2D3 was ineffective in a non-EMT model of resistance. We conclude that vitamin D sufficiency portends increased PFS among EGFR-mutant LUAD patients that receive EGFR TKIs, and that vitamin D signaling maintains drug efficacy in this specific patient subset by opposing EMT.
\end{abstract}

Keywords: lung cancer; EGFR; tyrosine kinase inhibitor; vitamin D; epithelial-mesenchymal transition

\section{Introduction}

Lung cancer is the most frequent cause of cancer-related mortality in the US and worldwide [1]. Treatment selection for lung cancer patients is determined by histologic and molecular features of the 
tumor. Activating mutations in the tyrosine kinase domain of the epidermal growth factor receptor (EGFR) gene, including exon 19 deletions (Del19) and exon 21 point mutations (L858R), drive lung tumorigenesis. Such molecular alterations occur in approximately $15 \%$ of US and $40 \%$ of Asian non-small cell lung cancer (NSCLC) cases [2,3]. Patients with advanced EGFR-mutant NSCLC are treated with EGFR tyrosine kinase inhibitors (EGFR TKIs) such as erlotinib (1st generation), afatinib (2nd generation), and osimertinib (3rd generation). In the majority of patients, the clinical course of EGFR TKI anti-tumor activity is characterized by an initial period of efficacy, followed by disease recurrence. There is a critical need to develop strategies to overcome resistance or extend the efficacy of TKIs in this patient population.

Acquired resistance to EGFR TKIs is frequently accompanied by epithelial-to-mesenchymal transition (EMT). This phenomenon is observed not only in preclinical models but also in clinical specimens [4-7]. Hence, the addition of pro-epithelial agents to the standard treatment of EGFR-mutant NSCLC may not only extend the duration of initial response to TKIs but also improve their efficacy after malignant progression. Vitamin D, a secosteroid hormone recognized for its role in muscular-skeletal and immune physiology, is capable of promoting apoptosis and epithelial differentiation in a variety of preclinical tumor models [8-12]. The link between vitamin D status and lung cancer in the clinical setting remains controversial. Several retrospective studies found an association between low serum 25-hydroxyvitamin D3 (25(OH)D3), a commonly used marker of vitamin D status, and increased risk of lung cancer mortality [13,14], while others found no such correlation [15-17]. A recently conducted prospective, double-blind placebo-controlled trial of vitamin D supplementation in unselected NSCLC patients demonstrated that supplementation provided no significant survival benefit in the overall cohort [18]. However, the vitamin D deficient subgroup of patients with early-stage LUAD that received vitamin D3 (1200 IU per day) versus placebo had significantly longer progression-free survival (PFS) and overall survival (OS). Notably, this trial was conducted in Japan, where EGFR mutations are the predominant oncogenic driver of LUAD.

In this regard, our previous work demonstrated that EGFR-mutant lung tumor models are uniquely sensitive to treatment with the active metabolite of vitamin D, 1,25-dihydroxyvitamin D3 $(1,25(\mathrm{OH}) 2 \mathrm{D} 3)$. This is largely due to their high expression of vitamin D receptor (VDR) and low levels of a key vitamin D catabolizing enzyme 24-hydroxylase, encoded by the CYP24A1 gene [19,20]. On the other hand, KRAS-mutant NSCLCs appear to have a VDR-low/CYP24A1-high expression pattern and are intrinsically refractory to $1,25(\mathrm{OH}) 2 \mathrm{D} 3$ treatment $[19,20]$. Cumulatively, these data raise the intriguing possibility that genotype-dependent differences in vitamin D3 signaling capacity exist and that it might be possible to exploit vitamin D3 supplementation preferentially in certain molecular subsets of LUAD to prevent disease progression. The benefits of vitamin D in molecularly defined subtypes of lung cancer have not been previously studied. Driven by the hypothesis that the benefits of vitamin $\mathrm{D}$ in patients is likely to be critically influenced by the molecular subtype of LUAD, in the current study we aim to investigate the prognostic value of vitamin D status in EGFR-mutant and KRAS-mutant NSCLC, as well as its activity in EGFR mutant LUAD cells (a candidate responsive subset).

\section{Results}

\subsection{Serum 25(OH)D3 Predicts PFS in EGFR-Mutant LUAD but Not KRAS-Mutant LUAD}

To evaluate the association between vitamin D serum concentrations and outcomes in molecularly defined LUAD subtypes, we retrospectively analyzed progression-free survival (PFS) in patients with advanced LUAD, whose tumors harbored either KRAS $(\mathrm{n}=34)$ or EGFR $(\mathrm{n}=21)$ mutations. Patient characteristics are shown in Table 1 and were balanced across tumor genotypes. All EGFR-mutant patients received first-line, standard of care treatment with EGFR TKIs (erlotinib $(n=20)$ or afatinib ( $n$ $=1)$ ). Patients with KRAS-mutant LUAD received a platinum-containing regimen as first-line therapy. For survival analysis, participants were divided into groups based on their serum 25(OH)D3 levels, with 
the cut-off set at $30 \mathrm{ng} / \mathrm{mL}$ based on Endocrine Society guidelines [21]. Then, $65 \%$ of the KRAS-mutant patients (22/34) and $62 \%$ of the EGFR-mutant patients (13/21) were classified as vitamin D-insufficient using this cut-off. A trend towards longer PFS in the $25(\mathrm{OH}) \mathrm{D} 3>30$ vs. $25(\mathrm{OH}) \mathrm{D} 3<30 \mathrm{ng} / \mathrm{mL}$ groups was detected in the total study population (KRAS-mutant and EGFR-mutant combined). However, statistical significance was not reached (10.5 months vs. 8.9 months in $25(\mathrm{OH}) \mathrm{D} 3>30 \mathrm{vs} .<30 \mathrm{ng} / \mathrm{mL}$, $p=0.056$; Figure 1A). We then performed a survival analysis in KRAS-mutant and EGFR-mutant subsets separately. High serum 25(OH)D3 did not provide PFS benefit in KRAS-mutant patients (6.2 vs. 6.0 months in 25(OH)D3 $>30$ vs. $<30 \mathrm{ng} / \mathrm{mL}, p=0.839$; Figure 1B). Since our previous work determined that vitamin D signaling is compromised in KRAS-mutant models of NSCLC, lack of association between 25(OH)D3 and PFS in this subset of patients was not unexpected [19]. Conversely, serum levels of 25(OH)D3 $>30 \mathrm{ng} / \mathrm{mL}$ predicted significantly longer PFS in the EGFR-mutant LUAD patients (14.5 vs. 10.6 months in $25(\mathrm{OH}) \mathrm{D} 3>30$ vs. $<30 \mathrm{ng} / \mathrm{mL}, p=0.022$; Figure $1 \mathrm{C}$ ). At the time of disease progression, when possible, the EGFR T790M gatekeeper mutation was screened for as a mechanism of EGFR TKI resistance. The T790M mutation was detected in $38.5 \%$ of vitamin D-insufficient and $37.5 \%$ of vitamin D-sufficient patients. T790M was tested for but not detected in $53.8 \%$ of vitamin D-insufficient and 37.5\% of vitamin D-sufficient patients. Material was not available for testing in $7.7 \%$ of vitamin D-insufficient and $37.5 \%$ of vitamin D-sufficient patients.

Table 1. Patients' characteristics. Tumor stage and patients' demographic characteristics were balanced across tumor genotypes.

\begin{tabular}{ccc}
\hline Characteristic & EGFR $^{\mathbf{m t}}(\mathbf{n}=\mathbf{2 1})$ & KRAS $^{\mathbf{m t}}(\mathbf{n}=\mathbf{3 4})$ \\
\hline Age (y), mean (SD) & $68(9)$ & $65(9)$ \\
Female, No. (\%) & $14(64)$ & $19(56)$ \\
African American, No. (\%) & $2(10)$ & $3(9)$ \\
Stage, No. (\%) & & \\
IV & $21(100)$ & $34(100)$ \\
Serum 25(OH)D3 (ng/ml), mean (SD) & $28.78(11.13)$ & $24.84(12.27)$ \\
\hline
\end{tabular}

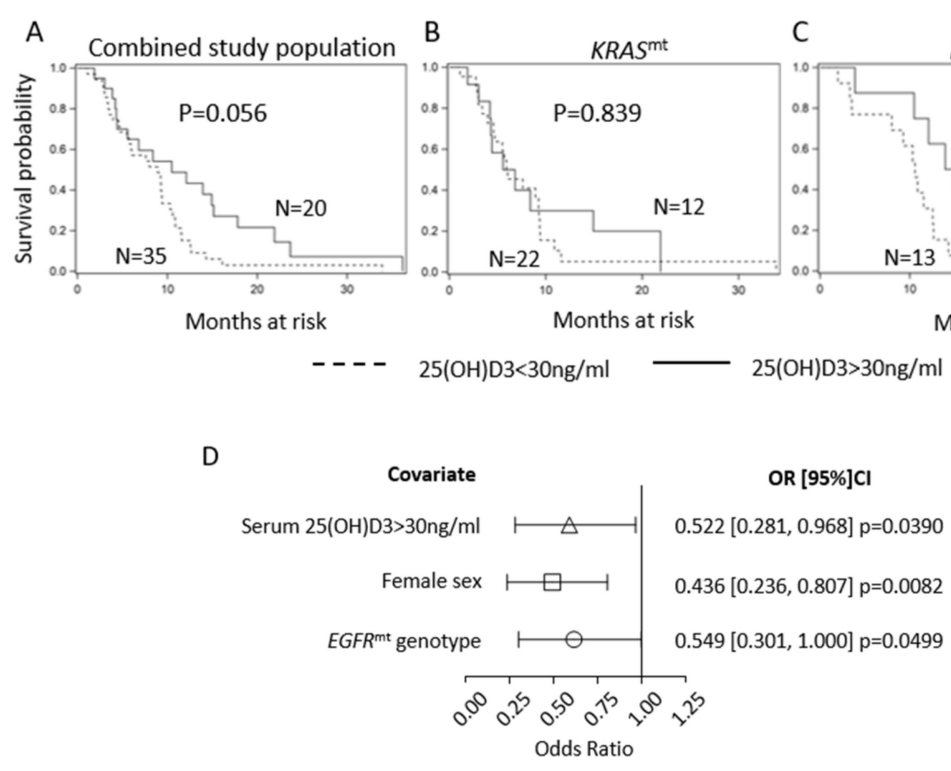

Figure 1. Serum 25(OH)D3 predicts PFS in EGFR-mutant LUAD but not KRAS-mutant LUAD. Participants were divided into groups based on their serum 25(OH)D3 levels with the cut-off set at $30 \mathrm{ng} / \mathrm{mL}$. Kaplan-Meier progression-free survival (PFS) analyses in combined EGFR- and KRAS-mutant cohort (A), KRAS-mutant (B), and EGFR-mutant (C) subsets are shown. (D) Forest plot of associations between PFS and serum 25(OH)D3/patient sex/tumor genotype. 
Additionally, we found a significant association between PFS and tumor genotype, patient sex, and vitamin D status, where EGFR mutation, female sex, and $25(\mathrm{OH}) \mathrm{D} 3>30 \mathrm{ng} / \mathrm{mL}$ directly associated with PFS (Figure 1D). Each of these three factors represented a relative risk reduction in PFS of roughly one half for the reference group relative to the non-reference group. Overall, the above findings suggest that vitamin D may provide survival benefit in a specific, molecularly defined subtype of NSCLC, namely EGFR-mutant LUAD.

\subsection{EGFR TKI Resistance Is Associated with EMT}

One possible explanation for the association between 25(OH)D3 sufficiency and longer benefit from early generation EGFR TKIs is that vitamin D signaling opposes EGFR TKI resistance. To test this idea, we investigated vitamin D receptor-mediated signaling, transcription, and function in models of acquired resistance to EGFR TKIs.

EGFR-mutant NSCLC cells H1975, SH416, and PC9 were subjected to chronic treatment with 0.5 $\mu \mathrm{M}$ osimertinib (H1975) or $1 \mu \mathrm{M}$ erlotinib (SH416 and PC9) to generate EGFR TKI-resistant sub-lines, H1975-OR, SH416-ER, and PC9-ER. IC50 and IC80 values for the TKIs were significantly increased in resistant cells as compared to parental counterparts (IC80 values of 14.2 vs. 3010, 11.3 vs. 2248, and 20.9 vs. 27,700 nM for H1975, SH416, and PC9 parental vs. resistant counterparts, respectively; Figure 2A). The emergence of additional mutations in the tyrosine kinase domain of the EGFR gene is the most prevalent mechanism of acquired resistance to EGFR TKIs $[22,23]$. To determine whether EGFR TKI resistance in our models was brought about by second or third site EGFR mutations, we sequenced exons 19-21 of the receptor. No additional mutations were found, indicating that the cells developed resistance by an EGFR-independent mechanism (Table S1).

Activation of bypass signaling pathways is another common mechanism of EGFR TKI failure [7,24-27]. In sensitive cells, EGFR TKIs block phosphorylation of EGFR and its downstream signaling molecules, such as AKT and ERK1/2, resulting in profound cell growth inhibition. However, in cells that acquire bypass-related resistance, these effector cascades remain activated and provide pro-survival signaling even in the presence of EGFR TKIs. To investigate the activation of bypass signaling in our EGFR TKI-resistant models, we examined phosphorylation of AKT and ERK1/2 in the absence and presence of EGFR TKIs. In contrast to SH416 and PC9 parental cells, erlotinib-resistant SH416-ER and PC9-ER cells sustained high levels of either pAKT (SH416-ER) or pERK1/2 (PC9-ER) after treatment with erlotinib. In SH416 parental cells, pAKT was undetected. In SH416-ER cells, pAKT was dramatically upregulated and only modestly reduced by erlotinib, despite complete pEGFR inhibition. Therefore, we conclude that both PC9-ER and SH416-ER cells developed EGFR TKI resistance associated with the activation of a bypass pathway (Figure 2B). Conversely, the effect of osimertinib on pERK1/2 and pAKT was similar in both H1975 parental and H1975-OR cells, suggesting a bypass-independent resistance mechanism (Figure 2B).

Upon microscopic examination, H1975-OR and SH416-ER, but not PC9-ER cells developed a spindle shape (Figure 2C), suggestive of EMT. Therefore, we examined expression of epithelial marker E-cadherin and mesenchymal marker vimentin in our models. We observed a striking loss of E-cadherin and gain of vimentin in H1975-OR and SH416-ER cells when compared to the corresponding parental lines (Figure 2D). Expression of additional epithelial and mesenchymal markers in H1975-OR cells is shown in Figure S1. These molecular changes were accompanied by a significant increase in invasion in H1975-OR and SH416-ER cells (2.9 and 1.5 times increase in invading fraction in H1975-OR and SH416-ER cells compared to corresponding parental counterparts; Figure 2E). In contrast, expression of E-cadherin and vimentin was similar in PC9 and PC9-ER cells Figure 2D), with PC9-ER cells having a lower invasion potential than their parental counterparts $(0.52$ relative invading fraction in PC9-ER compared to PC9 parental cells; Figure 2E). Similar results were previously reported by others: PC9 cells with acquired resistance to osimertinib maintained epithelial phenotype, while H1975 osimertinib-resistant cells developed mesenchymal features [28-30]. Overall, two out of three EGFR TKI-resistant models we developed (H1975-OR and SH416-ER) underwent EMT. Bypass activation was 
detected in PC9-ER and SH416-ER cells. Such heterogeneity is not unexpected and is representative of a diverse spectrum of therapy resistance mechanisms in the clinic.

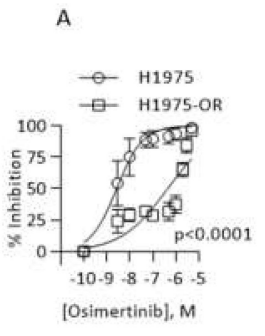

B

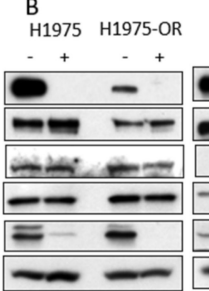

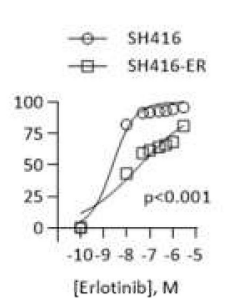

[Erlotinib], M

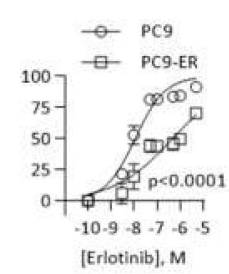

PC9-ER

\section{C}

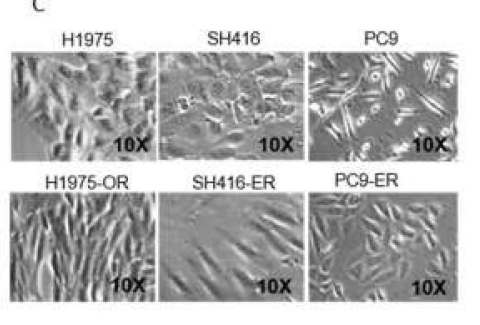

D

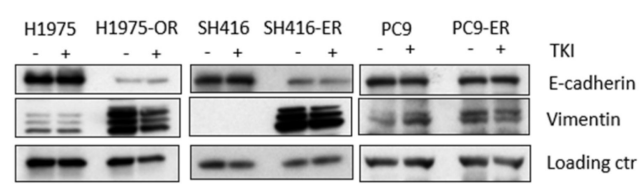

Figure 2. Prolonged exposure to EGFR tyrosine kinase inhibitors (EGFR TKIs) produces cells with different mechanisms of EGFR TKI-resistance. (A) H1975 cells were treated with $0.5 \mu \mathrm{M}$ osimertinib every $72 \mathrm{~h}$ for at least 30 days to produce H1975-OR cells. SH416 and PC9 cells were treated with $1 \mu \mathrm{M}$ erlotinib every $72 \mathrm{~h}$ for at least 30 days to produce SH416-ER and PC9-ER cells. Dose-response studies were conducted to determine IC50 and IC 80 values for parental cells and their established EGFR TKI-resistant derivatives. Drug response was measured after 9 days of treatment using sulforhodamine B (SRB) assay. The results shown for each model are the average (+/-SD) from 3 independent experiments. (B) The indicated cell lines were left untreated (-) or treated (+) with $0.5 \mu \mathrm{M}$ osimertinib (H1975 and H1975-OR) or $1 \mu \mathrm{M}$ erlotinib (SH416, SH416-ER, PC9, PC9-ER) for $24 \mathrm{~h}$. Expression of EGFR and its downstream effector molecules were investigated by Western blot. Loading controls used: $\beta$-Actin for H1975/H1975-OR, PC9/PC9-ER; $\alpha$-tubulin for SH416/SH416-ER. Quantitative band analysis is shown in Table S2. (C) Images of individual models were captured by phase contrast microscopy. (D) Expression of E-cadherin and vimentin was investigated by Western blot as an indicator of EMT. Images are representative of three independent experiments. Loading controls used: $\beta$-Actin for H1975/H1975-OR, PC9/PC9-ER; $\alpha$-tubulin for SH416/SH416-ER. Quantitative band analysis is shown in Table S3. (E) Parental cells and their TKI-resistant derivatives were subjected to transwell invasion assay, as described in Methods. The results shown are pooled from 3 independent experiments per cell line. Values obtained for drug-resistant cells were normalized to the corresponding parental cell line (arbitrarily set to an invasion score of 1.0 and represented by the dotted line). ${ }^{*} p<0.05 ;{ }^{* *} p<0.01$; ${ }^{* * *} p<0.005$.

\subsection{VDR Expression and Signaling Are Maintained in EGFR TKI-Resistant Cells}

Vitamin D signaling is initiated when 1,25(OH)2D3 binds to the vitamin D receptor (VDR). Previously, we demonstrated that EGFR-mutant treatment-naïve NSCLC cells and tumors express VDR and are sensitive to 1,25(OH)2D3 [19,20]. However, 1,25(OH)2D3 signaling capacity in models of acquired resistance to EGFR TKIs, including those with mesenchymal phenotype, have not been reported. Hence, we sought to characterize the 1,25(OH)2D3 signaling axis in our models of EGFR TKI resistance. In vitro studies were done using the active metabolite of vitamin $\mathrm{D}, 1,25(\mathrm{OH}) 2 \mathrm{D} 3$. $1,25(\mathrm{OH}) 2 \mathrm{D} 3$ is produced in the kidney and other tissues upon hydroxylation of its circulating precursor, 25(OH)D3, by the CYP27B1-encoded, $1 \alpha$-hydroxylase [31]. Although tumor cells are known to express CYP27B1 at varying levels (and produce 1,25(OH)2D3), conventionally, in vitro studies of vitamin D activity utilize 1,25(OH)2D3 to avoid variation in local production across models.

As shown in Figure 3A, VDR protein levels were reduced in SH416-ER and PC9-ER, but not H1975-OR cells when compared to their parental counterparts. Nevertheless, treatment with $1,25(\mathrm{OH}) 2 \mathrm{D} 3$ resulted in expected stabilization and increased levels of the receptor. This effect persisted 
in the presence of EGFR TKIs in all three resistant cell lines. Furthermore, 1,25(OH)2D3 induced transcriptional responses, as demonstrated by increased mRNA levels of two well-characterized direct targets of VDR, CYP24A1 and CAMP. CYP24A1 was upregulated in all cell lines under investigation (Figure 3B), and CAMP was increased in SH416-ER and PC9-ER (Figure 3C). Therefore, 1,25(OH)2D3 maintains signaling capacity in EGFR-mutant, TKI resistant cells, and so may be engaged to combat disease progression.
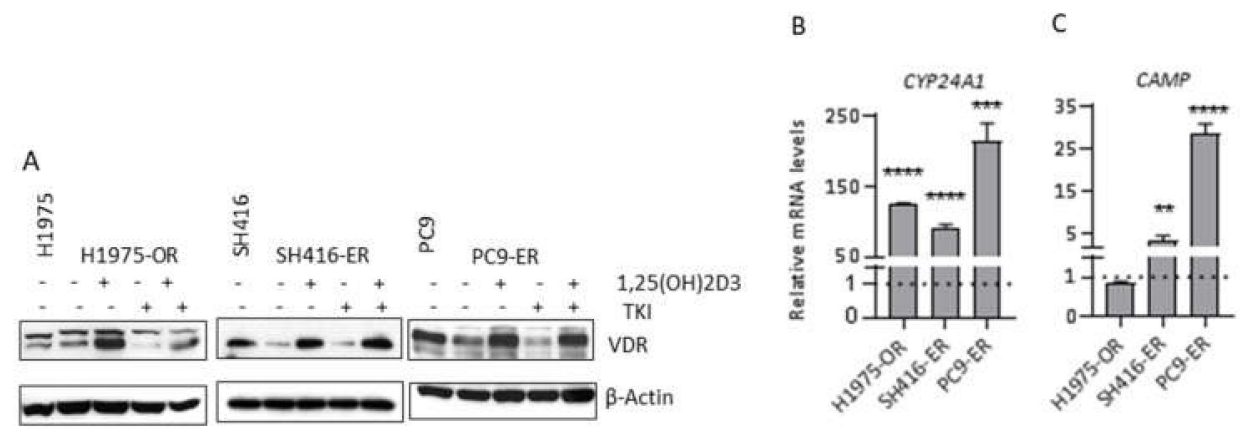

Figure 3. EGFR TKI resistant cells maintain 1,25(OH)2D3 signaling capacity. (A) EGFR TKI resistant cells were treated with vehicle control, $100 \mathrm{nM}$ 1,25(OH)2D3, $1 \mu$ M erlotinib (SH416-ER and PC9-ER), $0.5 \mu \mathrm{M}$ osimertinib (H1975-OR), or combination of 1,25(OH)2D3 and the corresponding EGFR TKI for $24 \mathrm{~h}$. (B,C) EGFR TKI-resistant cells were treated with $100 \mathrm{nM} 1,25(\mathrm{OH}) 2 \mathrm{D} 3$ for a total of $96 \mathrm{~h}$ and expression of VDR target genes was determined by qRT-PCR. ${ }^{* *} p<0.01$; ${ }^{* * *} p<0.001$; ${ }^{* * * *} p<0.0001$. Western blot analysis was performed at least twice with similar results. Representative images are shown. Quantitative band analysis is shown in Table S4. qRT-PCR was performed using RNA from three independent biological replicates for each cell line. Expression levels in vehicle control-treated cells is set to 1 and shown by the dotted line.

\subsection{1,25(OH)2D3 Promotes Epithelial Differentiation and Cell Cycle Arrest in EMT-Associated Models of EGFR TKI Resistance}

We previously reported the ability of 1,25(OH)2D3 to oppose TGF $\beta$-induced EMT in EGFR-mutant, TKI-naïve cells [32]. To investigate 1,25(OH)2D3 activity in EGFR TKI-resistant cells, we performed RNA sequencing of H1975 (Samples 1a, 1b, and 1c), H1975-OR (Samples 2a, 2b, and 2c), and H1975-OR cells treated with 1,25(OH)2D3 (Samples 3a, 3b, 3c). The H1975-based experimental models were selected for RNA sequencing because bypass activation did not contribute to their acquired EGFR TKI resistance. Hierarchical clustering and principal component analyses of the expression data identified a strong deviation from the norm and atypical gene expression pattern in Sample 3a (Figure S2). For these reasons we chose to exclude data obtained from Sample 3a from further analysis.

Based on the gene set enrichment analysis (GSEA), an enrichment map was constructed (Figure S3), which revealed that 1,25(OH)2D3 promoted epithelial differentiation in H1975-OR cells. The top 20 genes from the pro-epithelial signature identified by GSEA are shown in Figure 4A. The majority of these core enrichment genes were downregulated in the osimertinib-resistant model compared to the parental cells, while 1,25(OH)2D3 restored their expression in H1975-OR. Targeted qRT-PCR analysis confirmed statistically significant 1,25(OH)2D3-dependent induction of pro-epithelial CDH1 and CLDN4, and downregulation of pro-invasive MMP2 genes in both H1975-OR and SH416-ER cells (Figure 4B). On the protein level, we detected dramatic upregulation of E-cadherin in H1975-OR cells treated with 1,25(OH)2D3 even in the presence of osimertinib (Figure 4C). 
A

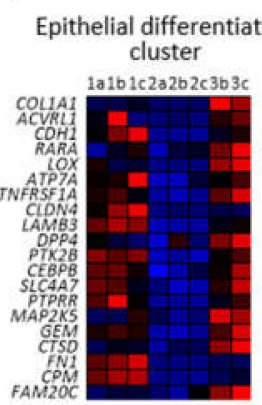

D

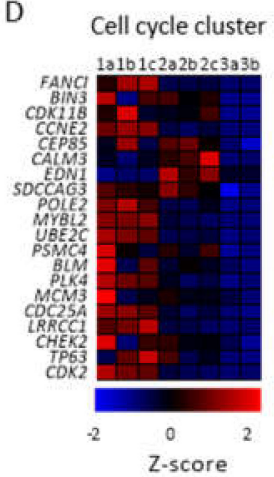

B

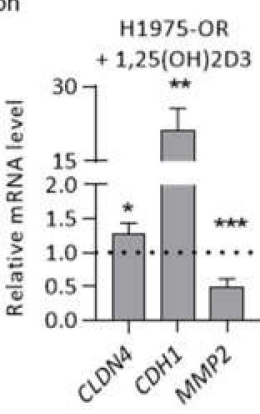

E
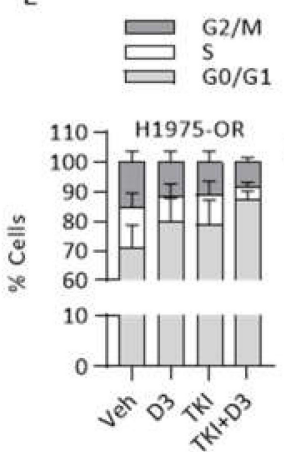

C
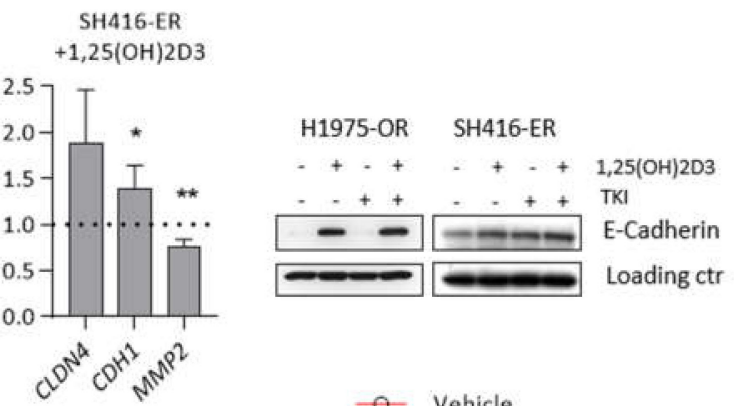

F

- - Vehicle

$\square \quad 1,25(\mathrm{OH}) 2 \mathrm{D} 3$

$\triangle$ Erlotinib

$\rightarrow$ Erlotinib $+1,25(\mathrm{OH}) 2 \mathrm{D} 3$
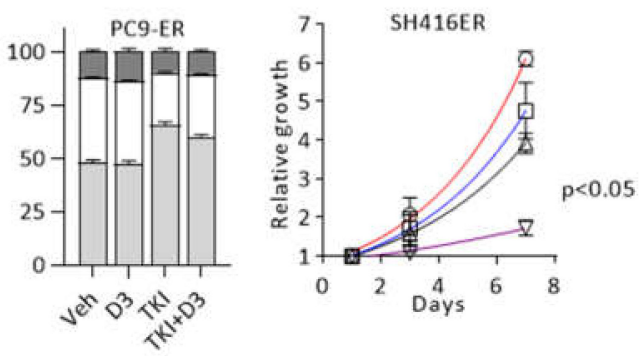

Figure 4. 1,25(OH)2D3 promotes epithelial differentiation and cell cycle arrest. RNA sequencing and GSEA were performed in H1975 parental (1a, 1b, 1c) and H1975-OR cells treated with vehicle control $(2 \mathrm{a}, 2 \mathrm{~b}, 2 \mathrm{c})$ or 1,25(OH)2D3 (3b, 3c). (A) Top 20 core enrichment genes from the Epithelial Differentiation cluster. (B). Expression of select epithelial and mesenchymal markers was investigated by qRT-PCR in H1975-OR and SH416-ER cells treated with 1,25(OH)2D3 for $96 \mathrm{~h}$. Expression level in vehicle-treated cells were set to 1 and are represented by the dotted line. ${ }^{*} p<0.05 ;{ }^{* *} p<0.01$; ${ }^{* * *} p<0.001$. (C) Cells were treated as indicated for $24 \mathrm{~h}$ and expression of E-cadherin was determined by immunoblot. Loading controls: $\beta$-Actin (H1975-OR) and $\alpha$-Tubulin (SH416-ER). Quantitative band analysis is shown in Table S5. (D) Top 20 core enrichment genes from Cell Cycle cluster. (E) Cell cycle distribution was analyzed by flow cytometry. Veh, vehicle control; D3, 1,25(OH)2D3; TKI, osimertinib (1975-OR) or erlotinib (PC9-ER). 1,25(OH)2D3 in combination with osimertinib significantly increased fraction of H1975-OR cells in the G0/G1 phase of the cell cycle (adj. $p<0.005$ vs. vehicle) but not in PC9-ER cells. (F) SH416-ER cells were treated as indicated and relative growth was estimated by SRB assay. All experiments were performed in triplicates.

RNA-sequencing also suggested that 1,25(OH)2D3 promoted cell cycle arrest in H1975-OR cells. The top 20 genes from the cell cycle signature are shown in Figure 4D. It is important to note that the majority of the top 20 cell cycle-related core enrichment genes were significantly downregulated in H1975-OR compared to the parental cells, signifying a prominent slow cycling phenotype associated with EGFR TKI resistance in this model. To validate the effect of 1,25(OH)2D3 on cell cycle distribution, we performed flow cytometric analysis in EGFR TKI resistant cells. 1,25(OH)2D3 in combination with EGFR TKI significantly increased the fraction of H1975-OR cells in G0/G1 phase (71\% vs. 87\% for vehicle and 1,25(OH)2D3 + TKI, respectively, $p<0.005$; Figure 4E). Conversely, 1,25(OH)2D3 did not significantly alter cell cycle distribution in EMT-independent PC9-ER cells (Figure 4E). Assaying cell cycle distribution by the means of flow cytometry in SH416-ER cells proved to be technically challenging due to aneuploidy, therefore, we opted to perform SRB-based doubling time analysis in this cell line. Although 1,25(OH)2D3 had little activity as a single agent, it significantly slowed doubling time when combined with erlotinib in SH416-ER cells compared to the vehicle treated cells (2.18 vs. 6.61 days in vehicle and 1,25(OH)2D3 + TKI, respectively, $p<0.05$; Figure 4F). Detailed statistical analysis of relative cell growth at day 7 in SH416-ER cells is provided in Table S6. The above results provide 
evidence that 1,25(OH)2D3 promotes epithelial differentiation and increases EGFR TKI-induced cell cycle arrest in EGFR-mutant EMT-associated models of acquired resistance to EGFR TKIs.

\subsection{1,25(OH)2D3 Promotes Sensitivity to EGFR TKIs in EMT-Associated Models of EGFR TKI Resistance}

Given the ability of 1,25(OH)2D3 to promote epithelial phenotype and deepen EGFR TKI-induced cell cycle arrest in H1975OR and SH416ER cells, we hypothesized that combining 1,25(OH)2D3 with EGFR TKIs in these models would result in improved growth suppression. To test this hypothesis, we first treated H1975-OR, SH416-ER, and PC9-ER cells with either vehicle control, 1,25(OH)2D3, a single concentration of EGFR TKI $(0.5 \mu \mathrm{M}$ osimertinib or $1 \mu \mathrm{M}$ erlotinib in corresponding cell lines), or the combination of 1,25(OH)2D3 and TKI. As a monotreatment, 1,25(OH)2D3 modestly suppressed the growth of EMT-associated H1975-OR and SH416-ER cells (Figure 5A). The greatest magnitude of growth suppression in the above cell lines was produced by the combination of EGFR TKIs and 1,25(OH)2D3 (79\% and 77\% growth inhibition in H1975-OR and SH416-ER cells treated with 1,25(OH)2D3 + TKI; Figure 5A). Consistent with the lack of cell cycle suppression observed in Figure 4E, 1,25(OH)2D3 alone or in combination with erlotinib did not impact the growth of PC9-ER cells (Figure 5A).

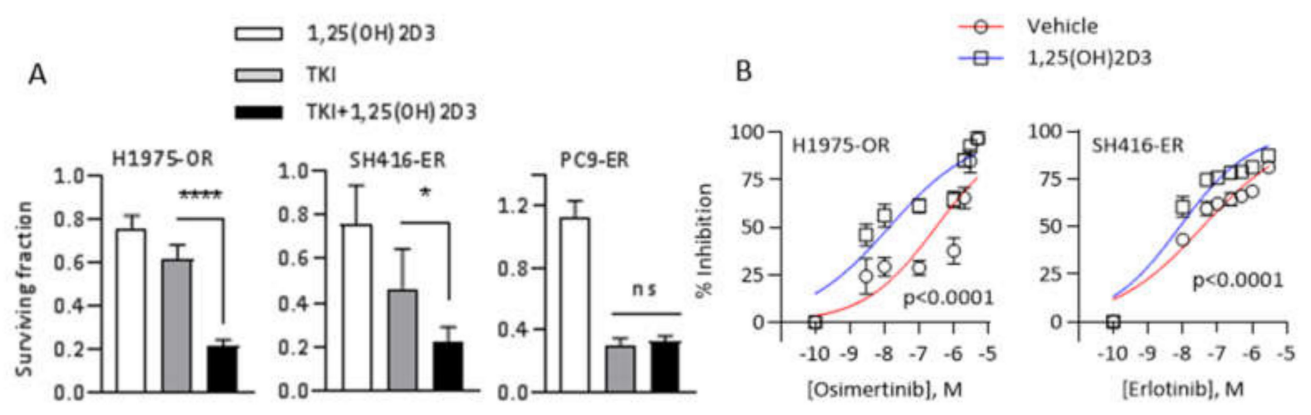

Figure 5. 1,25(OH)2D3 restores sensitivity to EGFR TKIs in EMT-associated models of acquired resistance to EGFR targeted therapies. (A) H1975, SH416, and PC9 cells and the corresponding TKI-resistant sublines were treated with vehicle control, $1 \mu \mathrm{M}$ erlotinib (SH416/SH416ER and PC9/PC9-ER) or $0.5 \mu \mathrm{M}$ osimertinib (H1975/H1975-OR), $100 \mathrm{nM}$ 1,25(OH)2D3, or combination of the above agents every $72 \mathrm{~h}$ for a total of 9 days. The number of surviving cells at day 9 in each treatment group was normalized to that in the vehicle control group. ${ }^{*} p<0.05 ;{ }^{* * * *} p<0.0001$. (B) H1975-OR and SH416-ER cells were treated with increasing concentrations of the corresponding EGFR TKIs alone or in combination with $1,25(\mathrm{OH}) 2 \mathrm{D} 3$ every $72 \mathrm{~h}$ for a total of 9 days. All experiments were performed in triplicates.

Next, we investigated the ability of 1,25(OH)2D3 to improve the efficacy of EGFR TKIs in EMT-associated models of EGFR TKI resistance. H1975-OR and SH416-ER cells were treated with increasing concentrations of osimertinib or erlotinib alone or in combination with $1,25(\mathrm{OH}) 2 \mathrm{D} 3$ and IC50/IC80 values of the TKIs were determined. Addition of 1,25(OH)2D3 significantly reduced concentrations of osimertinib and erlotinib necessary to produce $50 \%$ and $80 \%$ growth inhibition in both cell lines under investigation (IC80 values of $3010 \mathrm{nM}$ in vehicle-treated vs. $257 \mathrm{nM}$ in 1,25(OH)2D3-treated H1975-OR cells, and 2248 nM in vehicle-treated vs. $218 \mathrm{nM}$ in 1,25(OH)2D3-treated SH416-ER cells; Figure 5B). Additionally, 1,25(OH)2D3 had a similar effect on the efficacy of osimertinib in SH416-OR cells (Figure S4). These cells are osimertinib-resistant derivatives of the SH416 line that have previously been reported to undergo EMT as part of the resistance mechanism [27]. To test whether 1,25(OH)2D3 effects were specific to the drug-resistant state, we examined its interaction with osimertinib in parental H1975 cells. 1,25(OH)2D3 as mono-treatment was growth inhibitory in parental H1975 cells ( 20\% inhibition, Figure S5A). However, 1,25(OH)2D3 did not increase osimertinib sensitivity in this model (Figure S5B). We note that EGFR-mutant parental cells are intrinsically sensitive to EGFR inhibition. Therefore, it may be challenging to demonstrate further improvement in their 
response to TKIs in vitro. Overall, our results suggest that 1,25(OH)2D3 is capable of improving EGFR TKI efficacy, specifically in the EMT-associated models of acquired resistance to EGFR TKIs.

\section{Discussion}

Previous studies reported conflicting results regarding the link between serum vitamin D and survival in lung cancer patients [13-18]. However, the molecular subtypes of tumors have not been taken into consideration. Here, for the first time, we report that vitamin $\mathrm{D}$ activity may be restricted to a specific molecular subtype of lung cancer, adenocarcinomas harboring mutations in the EGFR gene. We found that high serum vitamin D predicted longer PFS in EGFR-mutant, but not in KRAS-mutant LUAD patients. In NSCLC, somatic alterations in the KRAS gene are reported to occur with at least double the frequency of those occurring in the EGFR $[33,34]$. Hence, in molecularly non-selected cohorts of patients, the survival benefit of vitamin D may not be apparent. Consistent with this interpretation, serum 25(OH)D3 status lost its prognostic value when analyzing our entire study cohort irrespective of genotype.

Overall, vitamin D insufficiency remains widely prevalent in lung cancer patients. We found that mean serum 25(OH)D3 levels in the total study cohort and in each of the molecularly-defined subsets was below $30 \mathrm{ng} / \mathrm{mL}$ in the majority of patients, indicating most patients had insufficient levels of vitamin D based on Endocrine Society guidelines [21]. Several factors may contribute to vitamin D insufficiency, including limited UVB exposure, smoking, and low levels of vitamin D in the diet. We note that some of the patients with EGFR mutant LUAD included in our study reported the use of vitamin D supplements. These individuals had higher levels of circulating 25(OH)D3 than those who did not report supplement use [35]. This observation is consistent with a role for variation in vitamin D intake as being determinative for serum 25(OH)D3 levels in patients with EGFR-mutant lung cancer.

Another implication of our data is that vitamin D supplementation may be of critical importance, particularly among lung cancer patients with EGFR mutations. We previously demonstrated that EGFR-mutant LUADs express significantly lower levels of CYP24A1 than KRAS-mutant cases [19]. CYP24A1 encodes the 24-hydroxylase that catabolically inactivates 1,25(OH)2D3. Although the mechanistic basis for this relationship remains unknown, variation in tumor 24-hydroxylase levels may contribute to a differential benefit of vitamin D supplementation in patients with KRAS-mutant and EGFR-mutant tumors.

It is worth noting that the results of our retrospective study are observational and not without limitations. We recognize that the sample size of the patient cohort is rather small, with only 21 EGFR-mutant patients. Despite this, the effect size was sufficiently large to detect a significant correlation. Further, our analysis determined a significant interaction between patient sex, tumor genotype, and PFS. Similar associations were previously reported in larger cohorts of lung cancer patients [36-40]. Additionally, the mean PFS in our EGFR-mutant subset of patients was 10.5 months; this, again, is comparable to the duration of response to erlotinib first-line therapy reported earlier [41]. Hence, our study cohort was not atypical compared to larger, previously studied LUAD patient cohorts.

Here, for the first time, we characterize the vitamin D signaling axes in EGFR TKI-resistant models. Previous reports indicate that VDR expression in lung tumors positively correlates with epithelial status [32]. Therefore, vitamin D activity may be compromised in EMT-associated resistance to EGFR TKIs. Here, we demonstrate that EGFR TKI-resistant cells, including those that underwent EMT, maintained VDR expression and are capable of mounting a transcriptional response to 1,25(OH)2D3. In these models, 1,25(OH)2D3 not only enhances the expression of epithelial markers but also induces cell cycle arrest and improves EGFR TKI efficacy. Moreover, we were able to generalize our findings across several cell models and EGFR TKIs. Due to our interest in EMT-associated resistance and the pro-epithelial actions of 1,25(OH)2D3, our current efforts are aimed at developing a clinically relevant orthotopic model of EGFR-mutant, TKI-resistant NSCLC. Utilizing such a model will allow us to interrogate not only the anti-growth but also the anti-metastatic potential of 1,25(OH)2D3 in vivo. 
$1,25(\mathrm{OH}) 2 \mathrm{D} 3$ is known to induce transcription of multiple cell adhesion molecules, including $C D H 1$ and a number of claudins [42,43]. Moreover, our earlier work showed that concurrent administration of 1,25(OH)2D3 and TGF $\beta$ in EGFR-mutant TKI-naïve cells opposed TGF $\beta$-driven EMT [32]. In the current study, we uncovered the ability of 1,25(OH)2D3 to support epithelial differentiation in the context of already established EMT. This is an important discovery since it points towards a potential opportunity to utilize vitamin D-based combination therapies in the setting of progressive disease. As a mechanism of resistance to EGFR TKIs, EMT is commonly observed in pre-clinical models [7,27-30,44]. In the clinic, EMT is believed to cause EGFR TKI failure in a small proportion ( 6\%) of EGFR-mutant LUAD patients [4]. However, the frequency of EMT-associated EGFR TKI resistance in the clinic may be greatly underestimated, as it is not commonly looked for. Intriguingly, in our study, cells that developed resistance to EGFR TKI erlotinib by EMT-independent mechanism (PC9-ER), despite preserved VDR expression and signaling, were refractory to the growth suppressive action of $1,25(\mathrm{OH}) 2 \mathrm{D} 3$. It is possible that the ability of 1,25(OH)2D3 to promote sensitivity to EGFR TKIs is linked to its pro-epithelial activity. On the other hand, according to the catalog of somatic mutations in cancer (COSMIC), PC9 cells harbor mutations in the genes encoding such key regulators of cell cycle progression as CCND2 and CDKN2A [45]. Mutations in these genes might have rendered 1,25(OH)2D3 inactive in this model. Further studies are needed to determine the exact link between EMT and vitamin D activity in EGFR TKI-resistant LUAD.

Another key observation presented here is that vitamin D status was not predictive of PFS in $K R A S$-mutant LUAD patients. As mentioned earlier, KRAS-mutant lung cancer cells are refractory to 1,25(OH)2D3 due to perturbations in key determinants of vitamin D signaling, such as low levels of VDR and elevated expression of CYP24A1 [19]. High local expression of CYP24A1 is predicted to limit tumor exposure to 1,25(OH)2D3, restricting its efficacy. While this hypothesis offers an explanation of why serum vitamin D levels were not associated with PFS in KRAS-mutant patients, it assumes that the major mechanism of action underlying vitamin D's efficacy is tumor-dependent (as opposed to "host"-dependent). In the retrospective study presented here, KRAS-mutation positive patients were treated with cytotoxic chemotherapies, whereas today, such patients may receive immune check point inhibitors. As vitamin D is known to play an important role in the immune physiology, vitamin D adequacy may still be uncovered as a prognostic factor in the context of the "host"-dependent mechanism of its action. In our ongoing investigations, we are looking to delineate the role of tumor CYP24A1 expression and activity in genotype- and therapy-specific responses to vitamin D.

In the era of precision medicine, careful selection of patients who may be intrinsically sensitive to the therapeutic agent is critically important. Hence, developing tailored vitamin D supplementation regimens in molecularly defined patient populations is critical to maximizing the utility of vitamin $\mathrm{D}$. Our results suggest that $E G F R$-mutant LUAD patients constitute a population with inherent sensitivity to vitamin $\mathrm{D}$ and therefore can derive a benefit from vitamin D-based combination treatments. We propose that further prospective studies aiming to delineate the role of vitamin $\mathrm{D}$ in the outcome of NSCLC should focus on the EGFR-mutant subset of this malignancy.

\section{Materials and Methods}

\subsection{Retrospective Clinical Study}

A retrospective, non-interventional biomarker study was conducted to examine the relationship between vitamin D status and progression-free survival in advanced LUAD patients. Analysis of the KRAS cohort described below was conducted with the approval of the Roswell Park IRB under non-human subjects research protocol \#BDR 117619. Analysis of the EGFR cohort was conducted with the approval of the Roswell Park IRB under non-human subjects research protocol \#BDR 091817.

To conduct the study, we first identified a set of patients diagnosed with advanced EGFR-mutant LUAD who had a serum sample in the Roswell Park Data Bank and BioRepository Shared Resource (DBBR), were treated with early generation EGFR TKIs (erlotinib $n=20$, afatinib $n=1$ ), and for whom 
outcome data were available. Next, KRAS cases were matched to EGFR cases based on patient age, sex, disease stage, and race resulting in cohorts with comparable demographics (Table 1). EGFR cases were from September 2010 to December 2016, KRAS cases were from January 2013 to March 2018. All cases included in the KRAS cohort received a regimen containing pemetrexed plus either cisplatin or carboplatin. Patients included in this case series were diagnosed prior to publicly available data showing overall survival benefit of first-line immunotherapy in combination with chemotherapy as a treatment option. EGFR and KRAS mutations were determined from genomic DNA obtained from tissue specimens subjected to PCR amplification and pyrosequencing to identify sequence variants for specimens tested prior to July 2014 (EGFR $\mathrm{n}=14, K R A S \mathrm{n}=14)$. All tissue specimen tests from 31 July 2014 onwards were based on next-generation sequencing technology $(E G F R \mathrm{n}=7$, KRAS $\mathrm{n}=20$ ). EGFR variants that were detected are shown in Table S7. De-identified serum samples for the cohort were requested from the DBBR and submitted to Heartland assays for the determination of 25-hydroxyvitamin D3 by gold-standard LC-MS/MS assay. Serum 25D3 levels were linked to annotated clinical information, and the data were submitted to the study statistician for analysis. Progression-free survival (PFS) was determined for each case and defined as time on first line therapy. Patients were classified as vitamin D3-sufficient $(\geq 30 \mathrm{ng} / \mathrm{mL} 25(\mathrm{OH}) \mathrm{D} 3)$ or vitamin D3-insufficient $(<30 \mathrm{ng} / \mathrm{mL})$ based on Endocrine Society guidelines [21]. In the EGFR cohort, two patients, one in each vitamin D arm, underwent EGFR TKI dose reduction.

\subsection{Cell Culture}

EGFR-mutant H1975 cells (EGFR T790M, L858R) were purchased from American Type Culture Collection (CRL-5908). EGFR-mutant PC9 lung adenocarcinoma cells (EGFR Del19) were generously provided by SI Abrams, PhD (Roswell Park Comprehensive Cancer Center, Buffalo, NY, USA). EGFR-mutant (EGFR Del19) SH416 cells were a gift from CM Lovly, MD, PhD (Vanderbilt University Medical Center, Nashville, TN, USA). H1975 and SH416 cells were cultured in RPMI 1640 (Corning, 1004-CV) supplemented with 10\% fetal bovine serum (FBS; Tissue Culture Biologicals 35-010-CV) and $1 \%$ penicillin-streptomycin (P/S; Corning, NY, 30-002-CI). PC9 cells were cultured in RPMI 1640 supplemented with 10\% FBS, 1\% P/S, 7.5\% HEPES (Corning, NY, 20-060CI), 1\% L-glutamine (Corning, NY, 25-005-CI), 1\% sodium pyruvate (Corning, NY, 25-000-CI), and 1\% MEM non-essential amino-acids (Corning, NY, 25-025-CI). EGFR TKI-resistant H1975-OR (osimertinib resistant), PC9-ER (erlotinib-resistant), and SH416-ER (erlotinib resistant) sublines were generated by culturing parental cells in growth media containing either $0.5 \mu \mathrm{M}$ osimertinib (S7297, Selleckchem, Houston, TX, USA) or $1 \mu \mathrm{M}$ erlotinib (OSI-774-01, OSI Pharmaceuticals, Long Island, New York) for $\geq 30$ days. Concentrations of the TKIs were chosen based on the reported steady state mean serum concentrations of the above drugs at the FDA-approved doses in patients [46,47]. Treatment media was replaced every $72 \mathrm{~h}$. Thereafter, EGFR TKI-resistant cell lines were continuously cultured in growth media containing $0.5 \mu \mathrm{M}$ osimertinib (H1975-OR) or $1 \mu \mathrm{M}$ erlotinib (PC9-ER and SH416-ER). Cells were maintained at $37^{\circ} \mathrm{C}$ and $5 \% \mathrm{CO}$. All cell lines routinely tested negatively for mycoplasma. EGFR mutations in parental cells and their drug resistant derivatives were verified through Sanger Sequencing using the protocol described by Molina-Vila et al. [48] (Table S1).

\subsection{EGFR TKI and 1,25(OH)2D3 Treatments}

1,25(OH)2D3 was kindly provided by Dr. Candace S. Johnson at Roswell Park Comprehensive Cancer Center, Buffalo, NY. For protein and RNA isolation, cells were plated in complete growth media at $40 \%-50 \%$ confluence. The next day, growth media was replaced with treatment media containing 10\% charcoal stripped serum (SH30068, HyClone, Pittsburgh, PA) and either vehicle control, EGFR TKI (erlotinib at $1 \mu \mathrm{M}$ or osimertinib at $0.5 \mu \mathrm{M}), 100 \mathrm{nM} 1,25(\mathrm{OH}) 2 \mathrm{D} 3$, or combination of the last two agents at the specified concentrations. For protein analysis, cells were treated for a total of $24 \mathrm{~h}$, with two treatments at time $=0 \mathrm{~h}(24 \mathrm{~h}$ after cell plating, referred to as $\mathrm{t} 0)$ and at $20 \mathrm{~h}$ time points $(4 \mathrm{~h}$ prior 
to cell harvest). For RNA analysis, cells were treated for a total of $96 \mathrm{~h}$, with treatments being applied at $\mathrm{t} 0,48$, and $92 \mathrm{~h}$ time points.

\subsection{Sulforhodamine B (SRB) Assay}

Sulforhodamine B (SRB) assay was widely used in this project to estimate cell numbers in a variety of experiments. The utility and methodology of the assay have been described previously [49]. Briefly, cells were fixed with 10\% trichloroacetic acid (TCA) (LC262301, LabChem, Zelienople, PA, USA) for $1 \mathrm{~h}$ at $4{ }^{\circ} \mathrm{C}$, washed, air dried, and stained with $0.57 \%(w / v)$ SRB dye (Sigma, S1402) in 1\% (v/v) acetic acid. The dye was solubilized in $10 \mathrm{mM}$ Tris base and the optical density (OD) at $510 \mathrm{~nm}$ was read on BioTek SYNERGY multimode microplate reader. Percent growth inhibition was calculated using the Equation (1):

$$
\% \text { inhibition }=\left(1-\frac{\text { absorbance vehicle }}{\text { absorbance treated }}\right) * 100 \%
$$

\subsection{IC50 and IC80 Determination}

Cells were plated in triplicate in complete growth media at $3 \times 10^{3}$ cells per well in 12-well plates and allowed to attach overnight. The next day, cells were treated with increasing concentrations of TKIs $(0-5 \mu \mathrm{M})$ alone or in combination with $100 \mathrm{nM} 1,25(\mathrm{OH}) 2 \mathrm{D} 3$ (as indicated for each experiment). Treatments were reapplied every $72 \mathrm{~h}$ for a total of 9 days to capture cytotoxic and cytostatic effects, followed by SRB assay.

\subsection{Cell Cycle Analysis}

Cells were fixed in cold $70 \%$ ethanol for a minimum of $30 \mathrm{~min}$. Cells were stained with $0.05 \mathrm{mg} / \mathrm{mL}$ propidium iodide (PI, P1304MP, Invitrogen, Waltham, MA, USA) in Krishan buffer $(0.1 \%$ sodium citrate, JT Baker, 3646-01; 0.02 mg/mL RNAse, Invitrogen, 12091-021; 0.2\% Igepal, Sigma I-3021; 1 drop of $1 \mathrm{~N} \mathrm{HCl}$ ). Samples were passed through a mesh filter (Corning, NY, \#352235), and PI fluorescence was detected by flow cytometry. Cell cycle distribution was determined using ModFit LT 4.0 software (Verity Software House, Topsham, ME, USA).

\subsection{Cell Doubling Time Determination}

Cells were plated at $3 \times 10^{3}$ cells/well in 12-well plates in triplicate, with a separate plate for each harvesting time point. Cells were harvested every $72 \mathrm{~h}$. Cell growth was determined by SRB assay. Only the exponential growth phase of each curve was considered for cell doubling time calculations. Relative growth is in reference to day one of the exponential growth phase. Doubling times were determined using exponential growth and non-linear regression analysis within GraphPad Prism v7.04 software.

\subsection{Transwell Invasion Assay}

Cells were plated at $3 \times 10^{3}$ cells per insert in Matrigel covered transwell invasion chambers (354480, Fisher Scientific, Waltham, MA, USA) in $100 \mu \mathrm{L}$ serum-free media. Then, $700 \mu \mathrm{L}$ of complete media was placed into the lower chamber. These were designated as invasion wells. Additionally, $3 \times 10^{3}$ cells were plated in $1 \mathrm{~mL}$ complete growth media in separate wells of the 24 -well plate without inserts (directly into the wells). These were designated as control wells. Then, $48 \mathrm{~h}$ after plating, cells were removed from the upper chamber of the invasion wells with a cotton swab. Cells in the control wells and the invasion wells were fixed in 10\% TCA and SRB assay performed to determine the cell number. Invasion was calculated using the following formulas:

$$
\frac{\text { absorbance invasion (migration) well }}{\text { absorbance control well }}=\text { invading fraction }
$$




$$
\frac{\text { invading (migrated) fraction experimental condition }}{\text { invading (migrated) fraction control condition }}=\text { normalized invasion }
$$

\subsection{RNA Isolation, $c D N A$ Synthesis, and $q R T-P C R$}

Cells were lysed in TRI-reagent (R2050-1-200, Zymo Research, Irvine, CA, USA) and RNA isolated using Direct-zol RNA miniprep kit (R2052, Zymo Research, Irvine, CA, USA). cDNA was synthesized using High Capacity cDNA Reverse Transcription Kit (Applied Biosystems, 4368814). The SYBR green/ROX/qPCR Master Mix kit (K0221, ThermoFisher Scientific, Waltham, MA, USA) and 7300 Real Time PCR System (Applied Biosystems, Beverly, MA, USA) were used for qRT-PCR reactions. Table S8 contains sequences of the qRT-PCR primers used in the study. Relative mRNA levels were determined using the $2^{-\Delta \Delta C t}$ method.

\subsection{Western Blot Antibodies and Detection Reagents}

Cell lysate preparation and Western blotting was performed using the standard lab protocol (20). The following primary antibodies were used for Western blotting: $\beta$-Actin (Santa Cruz Biotechnology, sc-1616), Akt (Cell Signaling Technology Danvers, MA, USA (CST), 9272), pAKT(Ser473) (CST, 9271), E-cadherin (BDTransduction Laboratories, San Jose, CA, USA (BD), 610181), EGFR (CST, 4405), pEGFR (Tyr1068) (CS, 2234), pMAPK (ERK1/2) (Thr202/Tyr204) (CST, 9101), $\alpha$-Tubulin (Millipore, 05-829), vimentin (BD, 550513). The following secondary antibodies were used: Anti-mouse IgG HRP-linked (NA931, GE Healthcare, Chicago IL, USA), anti-rabbit IgG HRP-linked (NA934, GE Healthcare, Chicago IL, USA), anti-rat IgG HRP-linked (NA935V, GE Healthcare, Chicago IL, USA). Bands were detected using Immobilon Crescendo Western HRP substrate (WBLUR0500, Millipore, Burlington, MA, USA). Quantitative band analysis was performed using Image Lab software (Bio Rad, CA, USA). Densitometry values are reported in Tables S5-S8.

\subsection{RNA Sequencing}

RNA was isolated from 3 separate passages of H1975 and H1975-OR cells treated with either

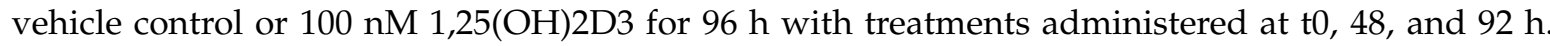
Sequencing libraries were prepared with the TruSeq Stranded Total RNA kit (20020596, Illumina Inc, San Diego, CA, USA), from 300 ng total RNA according to the manufacturer's instructions. The DNA libraries were quantitated using KAPA Biosystems qPCR kit and pooled together in an equimolar fashion. Each pool was denatured and diluted to $2.4 \mathrm{pM}$ with $1 \%$ PhiX control library (Illumina, FC110-3001) added. The resulting pools were loaded into the 150 cycle NextSeq high output Reagent cartridge (FC-404-2002, Illumina Inc, San Diego, CA, USA) for 75-cycle paired-end sequencing and sequenced on a NextSeq500 following the manufacturer's recommended protocol (Illumina Inc, San Diego, CA, USA). Data are available via the Gene Expression Omnibus (GEO) repository, accession GSE146850.

\subsection{Bioinformatics}

Raw FASTQ files were mapped onto the hg38 human genome using the Kallisto RNAseq quantification program [50], and gene level counts were obtained, summarizing transcript information with the TX import R package [51]. Gene set enrichment and leading edge analyses were performed using the Broad Institute GSEA 3.0 application [52]. RNA sequencing expression data were compared to the Gene Ontology (GO) dataset from molecular signature databases (MSigDB v6.2) [53]. An enrichment map was constructed using Cytoscape v3.6.1 software and the Enrichment map plugin [54]. Cytoscape Autoannotate application was used for gene set clustering, followed by manual curation of the clusters and annotations based on the GO categories (Figure S3). 


\subsection{Statistical Methods}

Patients' baseline characteristics (age, stage, and sex) and biomarker values in relation to progression-free survival were compared, and Cox proportional hazards regressions were used to evaluate associations in terms of the corresponding hazard ratios and 95\% confidence intervals. Graphical estimates of survival probabilities relative to progression-free survival were estimated using Kaplan-Meier estimators. All statistical tests were two-sided, and a $p$-value of $<0.05$ was considered statistically significant. All analyses were performed using SAS 9.4 (SAS Institute, Cary, NC, USA). The sample size was dictated by sample availability and was fixed at $n=55$.

Unless specified otherwise, in vitro experiments were performed in three biological replicates. Data are presented as mean \pm SD. Statistical analyses were performed in GraphPad Prism v7.04 software. Dose-response and doubling time studies were analyzed using non-linear regression. Non-linear models were fit to dose-response data and the LogIC50 and LogIC80 values were compared between the groups using an F-test. For relative mRNA expression and invasion assays, statistical significance was determined by two-sample $t$-tests. For the cell cycle distribution, statistical significance was calculated using 2-way ANOVA followed by Tukey's multiple comparison test. A $p$-value of $<0.05$ was considered statistically significant.

\section{Conclusions}

Vitamin D insufficiency is common among lung cancer patients. Although vitamin D has been shown to exert anti-tumor activity in multiple pre-clinical tumor models, its role in advanced lung cancer remains controversial. Here, for the first time, we investigated the prognostic value of vitamin D sufficiency in genotype-specific cohorts of advanced LUAD. Our results indicate that vitamin D has LUAD subtype-selective effects on clinical outcomes: EGFR-mutant patients derive a survival benefit from higher vitamin D levels, while KRAS-mutant patients do not. Mechanistically, vitamin D signaling promotes the expression of epithelial markers in EGFR-mutant cells and improves the efficacy of EGFR TKI treatment in vitro. Hence, vitamin D supplementation may be a safe and cost-effective tailored treatment approach for improving clinical outcomes in EGFR-mutant LUAD patients.

Supplementary Materials: The following are available online at http://www.mdpi.com/2072-6694/12/3/675/s1. Table S1. EGFR genotypes for the models used in the current study. Table S2. Western blot densitometry analysis related to Figure 2B. Table S3. Western blot densitometry analysis related to Figure 2D. Table S4. Western blot densitometry analysis related to Figure 3A. Table S5. Western blot densitometry analysis related to Figure 4C. Table S6. Statistical analysis of SH416-ER relative cell growth at day 7. Table S7. EGFR variants detected prior to therapy initiation in the EGFR-mutant cohort. Table S8. qRT-PCR primers and sequences. Figure S1. H1975-OR cells undergo EMT. Expression of epithelial (blue) and mesenchymal (red) markers was determined by qRT-PCR analysis. mRNA levels were normalized to those found in the parental H1975 cells (set to 1 and shown as the dotted line). ${ }^{* *}, \mathrm{p}<0.01 ;{ }^{* * *}, \mathrm{p}<0.001 ;{ }^{* * *}, \mathrm{p}<0.0001 ; \#, \mathrm{p}<0.00001 . \mathrm{N}=3$. Figure S2. Quality assessment analysis of the RNA sequencing results identified 3a sample as a deviation from the norm. Hierarchical clustering (A) and principal component analysis (B) revealed $3 a$ as an atypical sample that strongly deviated in gene expression pattern from other samples in the same treatment group, as well as other samples in the same cell line. Therefore, sample 3a was excluded from further gene set enrichment analysis. Figure S3. Enrichment map of upregulated (red) and downregulated (blue) GO categories in H1975-OR cells treated with 1,25(OH)2D3. Significantly enriched gene sets are represented by colored dots. Gene sets are grouped into clusters (circles) based on the description of ontological categories to illustrate up- and down-regulated biological processes in H1975-OR cells treated with 1,25(OH)2D3. Figure S4. SH416-OR cells were treated with increasing concentrations of osimertinib with or without 1,25(OH)2D3 every $72 \mathrm{~h}$ for a total of 9 days. Percent growth inhibition was determined by SRB assay. Significant decrease in osimertinib IC50 was found in the cells treated with 1,25(OH)2D3 (IC50 = 33.3 $\mathrm{nM})$ compared to those treated without $(\mathrm{IC} 50=217 \mathrm{nM}) . \mathrm{N}=3$. Figure S5. 1,25(OH)2D3 has no effect on EGFR TKI-sensitivity in H1975 parental cells. A. H1975 cells were treated as indicated every $72 \mathrm{~h}$ for a total of 9 days. Surviving cell fraction relative to vehicle control treated cells was determined by SRB assay. ${ }^{* * *}, p<0.0001$. B. H1975 cells were treated with increasing concentrations of osimertinib with or without $100 \mathrm{nM} 1,25(\mathrm{OH}) 2 \mathrm{D} 3$ every $72 \mathrm{~h}$ for a total of 9 days. Percent growth inhibition was determined by SRB assay.

Author Contributions: Conceptualization and experimental design, T.S., G.K.D., D.W.G., M.S., Y.Z., C.S.J., P.A.H. Methodology, T.S., A.H., S.B., G.K.D., P.A.H. Investigation, T.S., G.K.D., S.B., P.A.H. Formal analysis, T.S., S.B., A.H., P.A.H. Funding acquisition, M.S., D.W.G., P.A.H. Resources, G.K.D., C.M.L., C.S.J. Writing-original draft, T.S., G.K.D., A.H., P.A.H. Writing-review and editing, D.W.G., M.S., L.Z., C.M.L. Study supervision, P.A.H. All authors have read and agreed to the published version of the manuscript. 
Funding: This work was supported by the Roswell Park Comprehensive Cancer Center National Cancer Institute grant P30CA016056 involving the use of Roswell Park's DBBR, BDS, Genomic, and Biostatistics Shared Resources. The work was also generously supported by The Herd of Hope Award from the Roswell Park Alliance Foundation (to PAH, GKD, and DWG), NIH R03 CA249411 to PAH, and NIH R01 DE024595 to MS.

Acknowledgments: The authors wish to thank Suzanne Shoemaker for technical assistance with various aspects of this work.

Conflicts of Interest: The authors declare no conflicts of interest.

\section{References}

1. Didkowska, J.; Wojciechowska, U.; Manczuk, M.; Lobaszewski, J. Lung cancer epidemiology: Contemporary and future challenges worldwide. Ann. Transl. Med. 2016, 4, 150. [CrossRef] [PubMed]

2. Shigematsu, H.; Gazdar, A.F. Somatic mutations of epidermal growth factor receptor signaling pathway in lung cancers. Int. J. Cancer 2006, 118, 257-262. [CrossRef] [PubMed]

3. Shigematsu, H.; Takahashi, T.; Nomura, M.; Majmudar, K.; Suzuki, M.; Lee, H.; Wistuba, II; Fong, K.M.; Toyooka, S.; Shimizu, N.; et al. Somatic mutations of the HER2 kinase domain in lung adenocarcinomas. Cancer Res. 2005, 65, 1642-1646. [CrossRef] [PubMed]

4. Sequist, L.V.; Waltman, B.A.; Dias-Santagata, D.; Digumarthy, S.; Turke, A.B.; Fidias, P.; Bergethon, K.; Shaw, A.T.; Gettinger, S.; Cosper, A.K.; et al. Genotypic and histological evolution of lung cancers acquiring resistance to EGFR inhibitors. Sci. Transl. Med. 2011, 3, 75ra26. [CrossRef]

5. Chung, J.H.; Rho, J.K.; Xu, X.; Lee, J.S.; Yoon, H.I.; Lee, C.T.; Choi, Y.J.; Kim, H.R.; Kim, C.H.; Lee, J.C. Clinical and molecular evidences of epithelial to mesenchymal transition in acquired resistance to EGFR-TKIs. Lung Cancer 2011, 73, 176-182. [CrossRef] [PubMed]

6. Suda, K.; Tomizawa, K.; Fujii, M.; Murakami, H.; Osada, H.; Maehara, Y.; Yatabe, Y.; Sekido, Y.; Mitsudomi, T. Epithelial to mesenchymal transition in an epidermal growth factor receptor-mutant lung cancer cell line with acquired resistance to erlotinib. J. Thorac. Oncol. 2011, 6, 1152-1161. [CrossRef] [PubMed]

7. Zhang, Z.; Lee, J.C.; Lin, L.; Olivas, V.; Au, V.; LaFramboise, T.; Abdel-Rahman, M.; Wang, X.; Levine, A.D.; Rho, J.K.; et al. Activation of the AXL kinase causes resistance to EGFR-targeted therapy in lung cancer. Nat. Genet. 2012, 44, 852-860. [CrossRef] [PubMed]

8. Campbell, M.J.; Trump, D.L. Vitamin D Receptor Signaling and Cancer. Endocrinol. Metab. Clin. N. Am. 2017, 46, 1009-1038. [CrossRef] [PubMed]

9. Welsh, J.; Wietzke, J.A.; Zinser, G.M.; Smyczek, S.; Romu, S.; Tribble, E.; Welsh, J.C.; Byrne, B.; Narvaez, C.J. Impact of the Vitamin D3 receptor on growth-regulatory pathways in mammary gland and breast cancer. J. Steroid Biochem. Mol. Biol. 2002, 83, 85-92. [CrossRef]

10. Palmer, H.G.; Sanchez-Carbayo, M.; Ordonez-Moran, P.; Larriba, M.J.; Cordon-Cardo, C.; Munoz, A. Genetic signatures of differentiation induced by 1alpha,25-dihydroxyvitamin D3 in human colon cancer cells. Cancer Res. 2003, 63, 7799-7806.

11. Swami, S.; Krishnan, A.V.; Wang, J.Y.; Jensen, K.; Horst, R.; Albertelli, M.A.; Feldman, D. Dietary vitamin $\mathrm{D}(3)$ and 1,25-dihydroxyvitamin $\mathrm{D}(3)$ (calcitriol) exhibit equivalent anticancer activity in mouse xenograft models of breast and prostate cancer. Endocrinology 2012, 153, 2576-2587. [CrossRef] [PubMed]

12. Jeong, Y.; Swami, S.; Krishnan, A.V.; Williams, J.D.; Martin, S.; Horst, R.L.; Albertelli, M.A.; Feldman, B.J.; Feldman, D.; Diehn, M. Inhibition of Mouse Breast Tumor-Initiating Cells by Calcitriol and Dietary Vitamin D. Mol. Cancer Ther. 2015, 14, 1951-1961. [CrossRef] [PubMed]

13. Cheng, T.Y.; Neuhouser, M.L. Serum 25-hydroxyvitamin D, vitamin A, and lung cancer mortality in the US population: A potential nutrient-nutrient interaction. Cancer Causes Control 2012, 23, 1557-1565. [CrossRef] [PubMed]

14. Tretli, S.; Schwartz, G.G.; Torjesen, P.A.; Robsahm, T.E. Serum levels of 25-hydroxyvitamin D and survival in Norwegian patients with cancer of breast, colon, lung, and lymphoma: A population-based study. Cancer Causes Control 2012, 23, 363-370. [CrossRef]

15. Heist, R.S.; Zhou, W.; Wang, Z.; Liu, G.; Neuberg, D.; Su, L.; Asomaning, K.; Hollis, B.W.; Lynch, T.J.; Wain, J.C.; et al. Circulating 25-hydroxyvitamin D, VDR polymorphisms, and survival in advanced non-small-cell lung cancer. J. Clin. Oncol. 2008, 26, 5596-5602. [CrossRef] 
16. Vashi, P.G.; Edwin, P.; Popiel, B.; Gupta, D. The relationship between circulating 25-hydroxyvitamin D and survival in newly diagnosed advanced non-small-cell lung cancer. BMC Cancer 2015, 15, 1012. [CrossRef]

17. Anic, G.M.; Weinstein, S.J.; Mondul, A.M.; Mannisto, S.; Albanes, D. Serum vitamin D, vitamin D binding protein, and lung cancer survival. Lung Cancer 2014, 86, 297-303. [CrossRef]

18. Akiba, T.; Morikawa, T.; Odaka, M.; Nakada, T.; Kamiya, N.; Yamashita, M.; Yabe, M.; Inagaki, T.; Asano, H.; Mori, S.; et al. Vitamin D Supplementation and Survival of Patients with Non-small Cell Lung Cancer: A Randomized, Double-Blind, Placebo-Controlled Trial. Clin. Cancer Res. 2018, 24, 4089-4097. [CrossRef]

19. Zhang, Q.; Kanterewicz, B.; Shoemaker, S.; Hu, Q.; Liu, S.; Atwood, K.; Hershberger, P. Differential response to 1alpha,25-dihydroxyvitamin D3 (1alpha,25(OH)2D3) in non-small cell lung cancer cells with distinct oncogene mutations. J. Steroid Biochem. Mol. Biol. 2013, 136, 264-270. [CrossRef]

20. Verone-Boyle, A.R.; Shoemaker, S.; Attwood, K.; Morrison, C.D.; Makowski, A.J.; Battaglia, S.; Hershberger, P.A. Diet-derived 25-hydroxyvitamin D3 activates vitamin D receptor target gene expression and suppresses EGFR mutant non-small cell lung cancer growth in vitro and in vivo. Oncotarget 2016, 7, 995-1013. [CrossRef]

21. Holick, M.F.; Binkley, N.C.; Bischoff-Ferrari, H.A.; Gordon, C.M.; Hanley, D.A.; Heaney, R.P.; Murad, M.H.; Weaver, C.M.; Endocrine, S. Evaluation, treatment, and prevention of vitamin D deficiency: An Endocrine Society clinical practice guideline. J. Clin. Endocrinol. Metab. 2011, 96, 1911-1930. [CrossRef] [PubMed]

22. Yu, H.A.; Arcila, M.E.; Rekhtman, N.; Sima, C.S.; Zakowski, M.F.; Pao, W.; Kris, M.G.; Miller, V.A.; Ladanyi, M.; Riely, G.J. Analysis of tumor specimens at the time of acquired resistance to EGFR-TKI therapy in 155 patients with EGFR-mutant lung cancers. Clin. Cancer Res. 2013, 19, 2240-2247. [CrossRef] [PubMed]

23. Kobayashi, Y.; Togashi, Y.; Yatabe, Y.; Mizuuchi, H.; Jangchul, P.; Kondo, C.; Shimoji, M.; Sato, K.; Suda, K.; Tomizawa, K.; et al. EGFR Exon 18 Mutations in Lung Cancer: Molecular Predictors of Augmented Sensitivity to Afatinib or Neratinib as Compared with First- or Third-Generation TKIs. Clin. Cancer Res. 2015, 21, 5305-5313. [CrossRef] [PubMed]

24. Yano, S.; Wang, W.; Li, Q.; Matsumoto, K.; Sakurama, H.; Nakamura, T.; Ogino, H.; Kakiuchi, S.; Hanibuchi, M.; Nishioka, Y.; et al. Hepatocyte growth factor induces gefitinib resistance of lung adenocarcinoma with epidermal growth factor receptor-activating mutations. Cancer Res. 2008, 68, 9479-9487. [CrossRef]

25. Cortot, A.B.; Repellin, C.E.; Shimamura, T.; Capelletti, M.; Zejnullahu, K.; Ercan, D.; Christensen, J.G.; Wong, K.K.; Gray, N.S.; Janne, P.A. Resistance to irreversible EGF receptor tyrosine kinase inhibitors through a multistep mechanism involving the IGF1R pathway. Cancer Res. 2013, 73, 834-843. [CrossRef]

26. Shi, P.; Oh, Y.T.; Zhang, G.; Yao, W.; Yue, P.; Li, Y.; Kanteti, R.; Riehm, J.; Salgia, R.; Owonikoko, T.K.; et al. Met gene amplification and protein hyperactivation is a mechanism of resistance to both first and third generation EGFR inhibitors in lung cancer treatment. Cancer Lett. 2016, 380, 494-504. [CrossRef]

27. Ichihara, E.; Westover, D.; Meador, C.B.; Yan, Y.; Bauer, J.A.; Lu, P.; Ye, F.; Kulick, A.; de Stanchina, E.; McEwen, R.; et al. SFK/FAK Signaling Attenuates Osimertinib Efficacy in Both Drug-Sensitive and Drug-Resistant Models of EGFR-Mutant Lung Cancer. Cancer Res. 2017, 77, 2990-3000. [CrossRef]

28. Namba, K.; Shien, K.; Takahashi, Y.; Torigoe, H.; Sato, H.; Yoshioka, T.; Takeda, T.; Kurihara, E.; Ogoshi, Y.; Yamamoto, H.; et al. Activation of AXL as a Preclinical Acquired Resistance Mechanism Against Osimertinib Treatment in EGFR-Mutant Non-Small Cell Lung Cancer Cells. Mol. Cancer Res. 2019, 17, 499-507. [CrossRef]

29. Tang, Z.H.; Jiang, X.M.; Guo, X.; Fong, C.M.; Chen, X.; Lu, J.J. Characterization of osimertinib (AZD9291)-resistant non-small cell lung cancer NCI-H1975/OSIR cell line. Oncotarget 2016, 7, 81598-81610. [CrossRef]

30. Nukaga, S.; Yasuda, H.; Tsuchihara, K.; Hamamoto, J.; Masuzawa, K.; Kawada, I.; Naoki, K.; Matsumoto, S.; Mimaki, S.; Ikemura, S.; et al. Amplification of EGFR Wild-Type Alleles in Non-Small Cell Lung Cancer Cells Confers Acquired Resistance to Mutation-Selective EGFR Tyrosine Kinase Inhibitors. Cancer Res. 2017, 77, 2078-2089. [CrossRef]

31. Fraser, D.R.; Kodicek, E. Unique biosynthesis by kidney of a biological active vitamin D metabolite. Nature 1970, 228, 764-766. [CrossRef] [PubMed]

32. Upadhyay, S.K.; Verone, A.; Shoemaker, S.; Qin, M.; Liu, S.; Campbell, M.; Hershberger, P.A. 1,25-Dihydroxyvitamin D3 (1,25(OH)2D3) Signaling Capacity and the Epithelial-Mesenchymal Transition in Non-Small Cell Lung Cancer (NSCLC): Implications for Use of 1,25(OH)2D3 in NSCLC Treatment. Cancers (Basel) 2013, 5, 1504-1521. [CrossRef] [PubMed] 
33. Bauml, J.; Mick, R.; Zhang, Y.; Watt, C.D.; Vachani, A.; Aggarwal, C.; Evans, T.; Langer, C. Frequency of EGFR and KRAS mutations in patients with non small cell lung cancer by racial background: Do disparities exist? Lung Cancer 2013, 81, 347-353. [CrossRef]

34. Cancer Genome Atlas Research Network. Comprehensive molecular profiling of lung adenocarcinoma. Nature 2014, 511, 543-550. [CrossRef] [PubMed]

35. Shaurova, T.G.K.D.; Battaglia, S.; Hutson, A.; Hershberger, P.A. Unpublished Work; Roswell Park Comprehensive Cancer Center: Buffalo, NY, USA, 2019.

36. Chatkin, J.M.; Abreu, C.M.; Fritscher, C.C.; Wagner, M.B.; Pinto, J.A. Is there a gender difference in non-small cell lung cancer survival? Gend. Med. 2004, 1, 41-47. [CrossRef]

37. Sakurai, H.; Asamura, H.; Goya, T.; Eguchi, K.; Nakanishi, Y.; Sawabata, N.; Okumura, M.; Miyaoka, E.; Fujii, Y.; Japanese Joint Committee for Lung Cancer Registration. Survival differences by gender for resected non-small cell lung cancer: A retrospective analysis of 12,509 cases in a Japanese Lung Cancer Registry study. J. Thorac Oncol. 2010, 5, 1594-1601. [CrossRef] [PubMed]

38. Sagerup, C.M.; Smastuen, M.; Johannesen, T.B.; Helland, A.; Brustugun, O.T. Sex-specific trends in lung cancer incidence and survival: A population study of 40,118 cases. Thorax 2011, 66, 301-307. [CrossRef] [PubMed]

39. Wakelee, H.A.; Wang, W.; Schiller, J.H.; Langer, C.J.; Sandler, A.B.; Belani, C.P.; Johnson, D.H.; Eastern Cooperative Oncology Group. Survival differences by sex for patients with advanced non-small cell lung cancer on Eastern Cooperative Oncology Group trial 1594. J. Thorac Oncol. 2006, 1, 441-446. [CrossRef]

40. Sun, J.M.; Hwang, D.W.; Ahn, J.S.; Ahn, M.J.; Park, K. Prognostic and predictive value of KRAS mutations in advanced non-small cell lung cancer. PLoS ONE 2013, 8, e64816. [CrossRef] [PubMed]

41. Rosell, R.; Carcereny, E.; Gervais, R.; Vergnenegre, A.; Massuti, B.; Felip, E.; Palmero, R.; Garcia-Gomez, R.; Pallares, C.; Sanchez, J.M.; et al. Erlotinib versus standard chemotherapy as first-line treatment for European patients with advanced EGFR mutation-positive non-small-cell lung cancer (EURTAC): A multicentre, open-label, randomised phase 3 trial. Lancet Oncol. 2012, 13, 239-246. [CrossRef]

42. Palmer, H.G.; Gonzalez-Sancho, J.M.; Espada, J.; Berciano, M.T.; Puig, I.; Baulida, J.; Quintanilla, M.; Cano, A.; de Herreros, A.G.; Lafarga, M.; et al. Vitamin D(3) promotes the differentiation of colon carcinoma cells by the induction of E-cadherin and the inhibition of beta-catenin signaling. J. Cell Biol. 2001, 154, 369-387. [CrossRef] [PubMed]

43. Kong, J.; Zhang, Z.; Musch, M.W.; Ning, G.; Sun, J.; Hart, J.; Bissonnette, M.; Li, Y.C. Novel role of the vitamin D receptor in maintaining the integrity of the intestinal mucosal barrier. Am. J. Physiol. Gastrointest. Liver Physiol. 2008, 294, G208-G216. [CrossRef] [PubMed]

44. Hwang, W.; Chiu, Y.F.; Kuo, M.H.; Lee, K.L.; Lee, A.C.; Yu, C.C.; Chang, J.L.; Huang, W.C.; Hsiao, S.H.; Lin, S.E.; et al. Expression of Neuroendocrine Factor VGF in Lung Cancer Cells Confers Resistance to EGFR Kinase Inhibitors and Triggers Epithelial-to-Mesenchymal Transition. Cancer Res. 2017, 77, 3013-3026. [CrossRef]

45. Tate, J.G.; Bamford, S.; Jubb, H.C.; Sondka, Z.; Beare, D.M.; Bindal, N.; Boutselakis, H.; Cole, C.G.; Creatore, C.; Dawson, E.; et al. COSMIC: The Catalogue Of Somatic Mutations In Cancer. Nucleic Acids Res. 2019, 47, D941-D947. [CrossRef] [PubMed]

46. Dickinson, P.A.; Cantarini, M.V.; Collier, J.; Frewer, P.; Martin, S.; Pickup, K.; Ballard, P. Metabolic Disposition of Osimertinib in Rats, Dogs, and Humans: Insights into a Drug Designed to Bind Covalently to a Cysteine Residue of Epidermal Growth Factor Receptor. Drug Metab. Dispos. 2016, 44, 1201-1212. [CrossRef]

47. Hidalgo, M.; Siu, L.L.; Nemunaitis, J.; Rizzo, J.; Hammond, L.A.; Takimoto, C.; Eckhardt, S.G.; Tolcher, A.; Britten, C.D.; Denis, L.; et al. Phase I and pharmacologic study of OSI-774, an epidermal growth factor receptor tyrosine kinase inhibitor, in patients with advanced solid malignancies. J. Clin. Oncol. 2001, 19, 3267-3279. [CrossRef]

48. Molina-Vila, M.A.; Bertran-Alamillo, J.; Reguart, N.; Taron, M.; Castella, E.; Llatjos, M.; Costa, C.; Mayo, C.; Pradas, A.; Queralt, C.; et al. A sensitive method for detecting EGFR mutations in non-small cell lung cancer samples with few tumor cells. J. Thorac Oncol. 2008, 3, 1224-1235. [CrossRef]

49. Vichai, V.; Kirtikara, K. Sulforhodamine B colorimetric assay for cytotoxicity screening. Nat. Protoc. 2006, 1, 1112-1116. [CrossRef]

50. Bray, N.L.; Pimentel, H.; Melsted, P.; Pachter, L. Near-optimal probabilistic RNA-seq quantification. Nat. Biotechnol. 2016, 34, 525-527. [CrossRef] 
51. Soneson, C.; Love, M.I.; Robinson, M.D. Differential analyses for RNA-seq: Transcript-level estimates improve gene-level inferences. F1000Res 2015, 4, 1521. [CrossRef]

52. Subramanian, A.; Tamayo, P.; Mootha, V.K.; Mukherjee, S.; Ebert, B.L.; Gillette, M.A.; Paulovich, A.; Pomeroy, S.L.; Golub, T.R.; Lander, E.S.; et al. Gene set enrichment analysis: A knowledge-based approach for interpreting genome-wide expression profiles. Proc. Natl. Acad. Sci. USA 2005, 102, 15545-15550. [CrossRef] [PubMed]

53. Xie, X.; Lu, J.; Kulbokas, E.J.; Golub, T.R.; Mootha, V.; Lindblad-Toh, K.; Lander, E.S.; Kellis, M. Systematic discovery of regulatory motifs in human promoters and 3' UTRs by comparison of several mammals. Nature 2005, 434, 338-345. [CrossRef] [PubMed]

54. Merico, D.; Isserlin, R.; Stueker, O.; Emili, A.; Bader, G.D. Enrichment map: A network-based method for gene-set enrichment visualization and interpretation. PLoS ONE 2010, 5, e13984. [CrossRef] [PubMed]

(C) 2020 by the authors. Licensee MDPI, Basel, Switzerland. This article is an open access article distributed under the terms and conditions of the Creative Commons Attribution (CC BY) license (http://creativecommons.org/licenses/by/4.0/). 